\title{
Database Design on PE Network Selection Course System Guoquan Zhang ${ }^{1, a}$, Jingzhao Luan ${ }^{2, b}$ \\ ${ }^{1}$ Department of Physical Education, Bohai University, Jinzhou, P.R. China \\ ${ }^{2}$ State Grid Dalian Electric Power Supply Company, Dalian, P.R. China \\ a673722120@qq.com, b13889588669@139.com
}

Keywords: selection course information; concept design; E-R; model; database; logic design

\begin{abstract}
Course information database in selection course system occupies an important position, database design is an important part of software design, but also it is also the key supporting software system running, it is the starting point for software design, plays a decisive role in the qualitative change. In this paper, to conduct database design for PE network selection course system. System database mainly uses SQL Server 2008 database management tool design. First performed conceptual design, with E-R diagram shows a conceptual model; Then, introduced logic design, and its main job is to entity-relationship model (E-R diagram) derived from conceptual design into the relational model, on this basis, do proper optimization for it, each relationship is converted to table structure; and finally, discussed physical design and security design of the database.
\end{abstract}

\section{Introduction}

Database is the core and foundation of the selection course system, the selection course system in large amounts of data organized according to a certain data model, providing storage, maintenance, retrieve the data and other functions, so that the system can be convenient, timely and accurately obtain the required information from the database. The database is the key about the various components of selection course system closely together and how to combine. Database management system to manage and maintain the database through a data dictionary, is the core software of the database system. Database design is an important part of the system development and construction, for database designers, must have basic knowledge of databases and corresponding database design and technical knowledge, the use of software engineering principles and methods to achieve the design of the database [1]. Database construction is a combination of hardware and software, database design should be combined with the design of the system, to achieve the database behavioral design and structural design, while achieving the separation of the behavioral and structural design.

Database design is at a specified application environment, through constructed and optimized database physical structure and logical model, to establish a database application system, which should have the function to store and manage data, to meet the needs of the user's information applications, including data manipulation and course selection information management requirements [2]. Data manipulation refers to the operational requirements for data, such as query, add, delete, modify, and statistics; course selection information management requirements means to be stored and managed in a database to identify which data objects. Database design goal is to provide a sound infrastructure and efficient operating environment for users and application system. 


\section{Concept Design}

Database design tasks is for a given application environment, create a good database model, build databases and their application system, so that it can effectively collect, store, manipulate and manage data, to meet the various needs of users. Design content is mainly two aspects, includes the behavioral and structural characteristics design.

Elaborate conceptual structure design of the database, in the beginning of system design, the first consideration is how to use the data model to the real world objects abstract for structure and semantics of the database, namely conceptual structure design of database. Needs to be in the real world things are classified, clustering, generalization, establish an abstract conceptual data model, to reflect the structure of the data, relationships between data. This stage is necessary to avoid database implemented specifics details on computer, as far as possible in an abstract method to express [3]. Focus on conceptual design of the database as E-R diagram represents one of the ways that the entity attributes diagram for the abstract description of the real world, through the drawing tools of "boxes and arrows", through the analysis of user needs, establish a conceptual model of the database, to reflect the user's real environment and functional requirements, to help users build E-R diagrams to describe the specific structure of the data. E-R diagram consists of entities, attributes, and relation, the three parts to represent the relationship of the system. It is independent of the specific database management system, and how to implement the database should also irrelevant.

E-R diagram is proposed Entity-Relationship approach by P.P.S CHEN in 1976 [4]. In E-R diagram, E-R diagram has three elements, entities to indicate the name of the entity type is usually used a rectangular to represent, attribute use oval to represent, diamond said the relationships between the entity type, write relationship name in the diamond box, and are connected by an undirected edge with relevant entities type, while in undirected edges marked the type of relationship (1: 1, 1: $\mathrm{n}$ or $\mathrm{m}: \mathrm{n})$, can draw an E-R diagram. The E-R diagram of system is shown in Fig. 1.

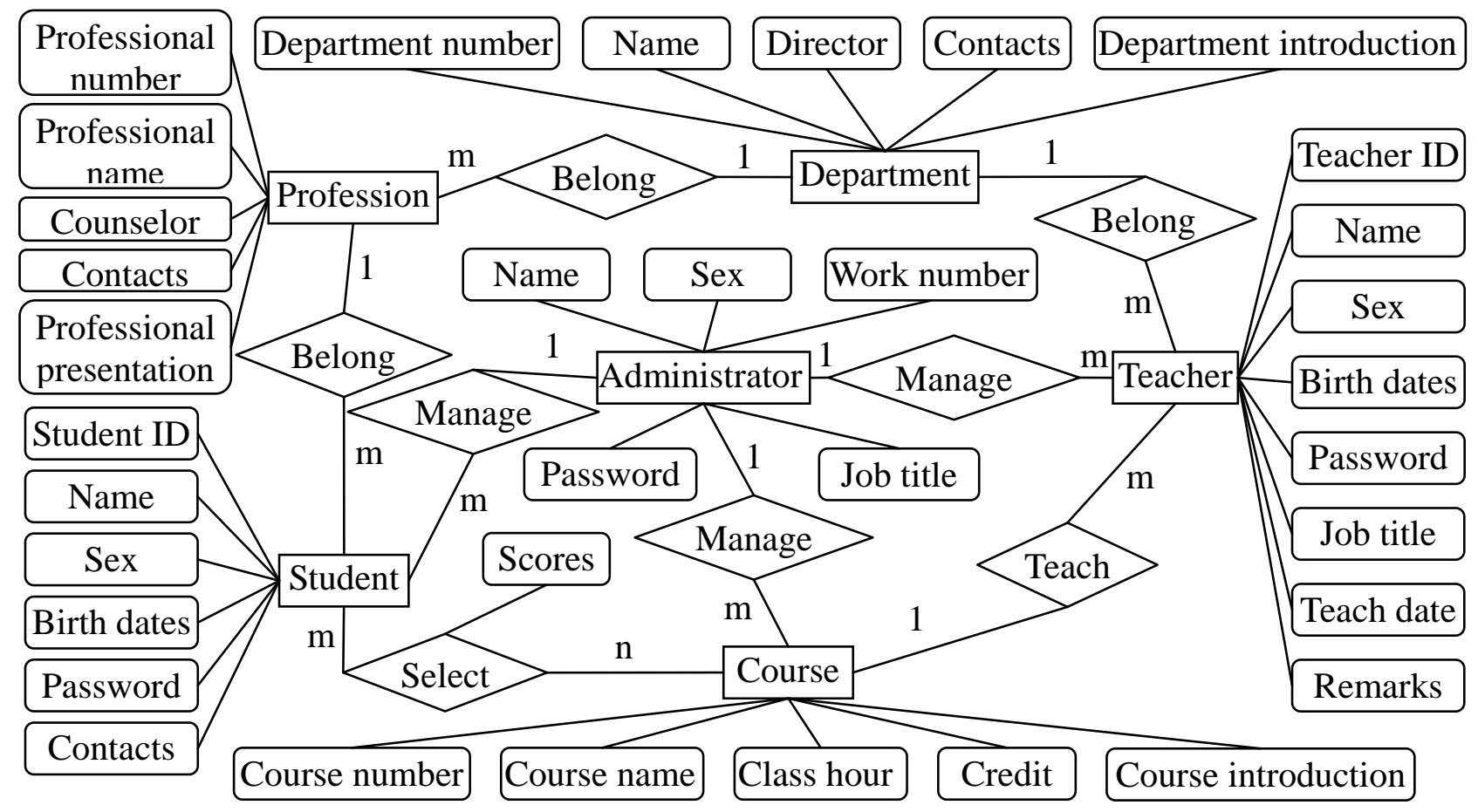

Fig. 1. The system E-R diagram 


\section{Logic Design}

Obtained a conceptual data model in the previous section, which is independent of the specific DBMS information model, database system implementation must be obtained conceptual data model be converted to the logic model of a specific DBMS products support, using Microsoft SQL Server 2008 database management system, therefore, the logical design is based on SQL Server 2008 performance characteristics, the E-R diagram model of conceptual design phase transfer the process for the logical model supported. Microsoft SQL Server 2008 is a distributed relational database management system by Microsoft Corp that provides data query, search, synchronize, report, and analyze, a series of rich integrated operation services, to provide efficient, easy to use, manageable data storage and data retrieval functions for other applications software [5].

In the process of the conceptual model is converted into a logical model, conceptual structure design phase of establishing the basic E-R diagram, according to the selected management system software to support data model (hierarchical, mesh, relationship), converted to the corresponding logical model. This conversion should comply with the principles of the relational data model. In order to further improve the performance of database, normalization theory can be used to modify, adjust the model structure of the database, determine data dependencies, eliminating redundant data, to achieve optimal data model. E-R diagram to convert the relational model is to solve how to contact between the entities and entities convert relationships, and determine the properties and codes of these relationships. This conversion generally based on the following principles [6]:

An entity converts a relationship, the attribute of entity is the attribute of relationships, the code of entity is also the code of relationship; A contact is also converted to a relationship, the attribute of relationship and contact associated entity codes are converted into the attribute of relationship, but the code of relationship will be changed according to the type of contact, if it is: 1: 1 contact, both ends of the entities code have become a candidate code of relationship; 1 : $n$ contact, $n$ side entity code becomes a relationship code; m: $n$ contact, both ends of the combined entity code becomes the relationship code.

The main work of logic design, entity-relationship model is derived from the conceptual design converted into a relational model. The details are as follows: Administrator (name, sex, job number, password, job title); Teacher (teacher ID, teacher name, sex, password, teacher titles, teaching date); Student (student number, student name, sex, birth date, password, contact details); Courses (course number, course name, class hours, course description); Department (department number, department name, department director, contact information, department introduction); Profession (professional number, professional name, counselor, contact information, professional presentation)

Then the establishment of a database table structure, on the basis of the relational model derived in the logical design phase, and its proper optimization, transformed each relationship converted into table structure, student course selection table structure as shown in Table 1:

Table 1. Student selection course table

\begin{tabular}{c|c|c|c|c|c|c|c}
\hline $\begin{array}{c}\text { Fields } \\
\text { NO }\end{array}$ & Fields Name & $\begin{array}{c}\text { Fields } \\
\text { Type }\end{array}$ & $\begin{array}{c}\text { Fields } \\
\text { Width }\end{array}$ & $\begin{array}{c}\text { Fields } \\
\text { NO }\end{array}$ & Fields Name & $\begin{array}{c}\text { Fields } \\
\text { Type }\end{array}$ & $\begin{array}{c}\text { Fields } \\
\text { Width }\end{array}$ \\
\hline 1 & StudentName & Char & 20 & 7 & CourseIn & Char & 50 \\
\hdashline 2 & StudentID & Char & 20 & 8 & CourseTeacher & Char & 20 \\
\hdashline 3 & StudentDepart & Char & 40 & 9 & TeacherNo & Char & 20 \\
\hdashline 4 & StudentSex & Char & 10 & 10 & ClassScore & Char & 10 \\
\hdashline 5 & CourseNo & Char & 20 & 11 & Credit & Char & 20 \\
\hdashline 6 & CourseName & Char & 20 & 12 & Remark & Varchar & 50 \\
\hline
\end{tabular}




\section{Physical Design and Security Design}

Physical design of database is considered on the basis of logic design how to meet the needs of data access, which includes design and create a reasonable index, use partitioning technology will be large tables broken down into small table, thus, use partitioning eliminate to satisfy database management and data access performance requirements, using tables compression to reduce storage space of data, design a database file storage policy balance I/O, reduce data competition [8].The core of physical design is to ensure efficient database access and data storage in database. For this core, the goal of physical design is to maximize should improve the performance about the database application program for database access and storage. Ultimately through the rational use of physical resources, these physical resources such as CPU, memory, I/O and network delay to achieve the purpose of optimizing the database physical design.

Database security design, network security is very important, from the database level, server level and application level into comprehensive consideration, in the course selection, according to different users, set different permissions, different initialization menu; In this system, the security of the database mainly through access control mechanisms implemented in the database, firstly defined some operating authority, namely the role of various management personnel, followed by the definition of the database login, finally according to user permissions table will log allocation for the appropriate roles, due to the complexity of the personnel, the role is divided into three levels: administrators, teachers, students, even when a teacher for the course additions and deletions to operate, does not affect the user's operation of other teachers, but only change the number of users in the roles, the establishment of a dedicated system permissions table to record the user and permissions, for different types of users and assign the appropriate permissions and account; administrator is the highest level, in order to further protect the database, create a backup database server, regular database backup and replication, system database mainly uses SQL Server 2008 database management tools for the design, it has strong management, security, login, network support functions, and can and other database conversion, is a network database management software, meets the design requirements [9].

\section{Conclusion}

Normalized database design is one of key to achieve scientific management for course selection data, the biggest advantage is that the database can be a lot of information organized in a certain structure, and can be centrally stored and managed in the system all the data in an orderly manner. Design and implementation of background database to avoid the arising security issues, efficiency issues due to network transfer. In this paper, to achieve the design of the database according to the principles and methods of software engineering, conducted a conceptual design, logical design, physical design and security design. Largely to meet the business needs of the application system, described the detail information about system database tables, and through the E-R model intuitive describes the attributes and related to each entity in the system database. Database design is provide a sound infrastructure and efficient operating environment for users and application system.

\section{References}

[1] H. Zhang, "The Design and Implementation of Hospital Information System," Chang'an University, 2013.

[2] Y. H. Yan, "The Design of the Hospital Information System Network and Database," Hebei North University, 2013.

[3] X. Sun, "The Design and Achievement of Online Course Selection System Based on JSP Technology," Tianjin Normal University, 2014.

[4] Z. M. Luo, "Design and Implementation of University Public Elective Courses Online Course Selection System," South China University of Technology, 2013. 
[5] Y. F. Lu, "Database Development of Computer Room Intelligent Monitoring and Management System," South China University of Technology, 2013.

[6] Baidu, "Logic Model," http://blog.sina.com.cn/s/blog_9e55c39001018b28.html, 2015-5-2.

[7] H. J. Liu, "The Design and Implementation of Database for Online Examination System," Computer Knowledge and Technology, vol. 8, no. 3, pp.508-511, 2012.

[8] S. Z. Lin, "The Research on Optimization of Physical Database Design of Information System," Tianjin University, 2012.

[9] Y. Wang, "The Database Design and Implementation of Research Project Management System," Computer Programming Skills and Maintenance, vol. 17, no. 8, pp.60-64, 2010. 